\title{
Dilatonic, Chiral Cosmic Strings
}

\author{
Vanessa C. Andrade \\ Departamento de Física, Univ. de Brasília, Brazil \\ E-mail: andradedfis.unb.br
}

\section{André Luiz Naves de Oliveira*}

Departamento de Física, Univ. de Brasília, Brazil

E-mail: andreodfis.unb.br

\section{Maria Emília X. Guimarães}

Departamento de Matemática, Univ. de Brasília, Brazil

E-mail: margeunb.br

\begin{abstract}
In this work, we deal with the chiral string model for which the world-sheet current is null in the framework of a scalar-tensor gravity. Our main goal is to analyse the impact of such a current on the gravitational macroscopic effects. For the purpose of this analysis, we first study the gravitational properties of the spacetime generated by this string in the presence of a dilaton field. Then, we carry out an investigation of the mechanism of formation and evolution of wakes in this framework, showing the explicit contribution of the chirality to this effect.
\end{abstract}

Fourth International Winter Conference on Mathematical Methods in Physics 09 - 13 August 2004

Centro Brasileiro de Pesquisas Fisicas (CBPF/MCT), Rio de Janeiro, Brazil

* Speaker. 


\section{Introduction}

The present work deals with the case of a chiral string model in the framework of a scalartensor gravity. In particular, we are interested in the macroscopic description of the effect in cosmic strings of mechanisms of the various (fermionic and bosonic superconducting) kinds originally proposed by Witten [1]. Whilst considering current-carrying cosmic vortex, we will also add scalartensor corrections on its metric. The motivation relies on the fact that theoretically, the possibility that gravity might not be fundamentally described by purely tensorial theory in four dimensions is growing in importance. This is in part a consequence of superstring theory, which is consistent in ten dimensions (or M-theory in eleven dimensions), but also the more phenomenological recent developments of "braneworld" scenarios [2, 3] have motivated the study of other gravitational theories in four-dimensions. In fact, the origin of the (gravitational) scalar field can be many: the scalar field arising from the size of the compactified internal space in the Kaluza-Klein theory; the zero mode (dilaton field) described by a symmetric second-rank tensor behaving as space-time metric at low energy level in the closed string theory; the scalar field in a brane world scenario; and more ${ }^{1}$. In any case, clearly, it is expected that both the small scale implications of alternative theories of gravity [5] and the large scale implications of modified gravity [6] of such scenarios have a direct impact for astrophysics and cosmology.

The combination of both conducting properties of a string and the modified gravity (in particular, the scalar-tensor models) is, of course, of real interest in astrophysics and cosmology and has been treated elsewhere [7, 8, 9]. The most noticeable effect was point out by Peter, Guimarães and Andrade [10] in which the (gravitational) scalar field ${ }^{2}$ behaves as a winding phase along the string, thereby generating a neutral current kind of effect by raising the degeneracy between the eigenvalues of the stress-energy tensor.

Our purpose in this paper is twofold. We first present the metric generated by a chiral cosmic string in the scalar-tensor gravity. This metric is obtained straightforwardly by an algorithm developed in the reference [11]. The second and main goal of this work is to explore how this null current - chirality - affects the motion of particles in this spacetime. Namely, we analyze the formation and evolution of wakes in this model. We anticipate that the main conclusion of this paper is to show that, up to first order in a parameter of the underlying theory, the mechanism of accretion is not sensitive to the "conducting properties" of this model, the result being qualitatively very similar to the mechanism by a neutral string.

This work is outlined as follows. In section 2, we present the gravitational field of a scalartensor chiral string and we consider the mechanism of formation and evolution of wakes in this framework by means of the Zel'dovich approximation. In section 3, we summarize the main conclusions and compare our results with the case of a neutral cosmic string in general relativity [12] and with the cases of a neutral [13] and a chiral [14] in scalar-tensor gravity.

\footnotetext{
${ }^{1}$ For a deeper discussion on this subject, we refer the reader to the book of Y. Fujii and K. Maeda [4].

${ }^{2}$ From now on, we will call this field generically as "dilaton".
} 


\section{The scalar-tensor chiral string and the wake formation}

\subsection{The gravitational field}

In this work, we will modify gravity within a class of scalar-tensor theories such that the action is written as:

$$
\mathcal{S}=\frac{1}{16 \pi} \int d^{4} x \sqrt{-\tilde{g}}\left[\tilde{R} \tilde{\Phi}-\frac{\omega(\tilde{\Phi})}{\tilde{\Phi}} \partial^{\mu} \tilde{\Phi} \partial_{\mu} \tilde{\Phi}\right]+\mathcal{S}_{m}\left[\Psi_{m}, \tilde{g}_{\mu v}\right],
$$

$\tilde{g}_{\mu \nu}$ is the physical metric which contains both scalar and tensor degrees of freedom, $\tilde{R}$ is the curvature scalar associated to it and $\mathcal{S}_{m}$ is the action for general matter fields which, by now, is left arbitrary.

By varying action (2.1) with respect to the metric $\tilde{g}_{\mu \nu}$ and to the scalar field $\tilde{\Phi}$ we obtain the modified Einstein equations and a wave equation for $\tilde{\Phi}$ :

$$
\begin{aligned}
\tilde{R}_{\mu v}-\frac{1}{2} \tilde{g}_{\mu v} \tilde{R}= & \frac{8 \pi}{\tilde{\Phi}} \tilde{T}_{\mu v}+\frac{\omega(\tilde{\Phi})}{\tilde{\Phi}}\left[\partial_{\mu} \tilde{\Phi} \partial_{v} \tilde{\Phi}-\frac{1}{2} \tilde{g}_{\mu \nu} \partial^{\alpha} \tilde{\Phi} \partial_{\alpha} \tilde{\Phi}\right] \\
& +\frac{1}{\tilde{\Phi}}\left(\nabla_{v} \tilde{\Phi}_{, \mu}-\tilde{g}_{\mu v} \square_{\tilde{g}} \tilde{\Phi}\right) \\
\square_{\tilde{g}} \tilde{\Phi}= & \frac{1}{2 \omega(\tilde{\Phi})+3}\left[8 \pi \tilde{T}-\frac{d \omega}{d \tilde{\Phi}} \partial_{\mu} \tilde{\Phi} \partial^{\mu} \tilde{\Phi}\right] \\
\nabla_{\mu} \tilde{T}_{v}^{\mu}= & 0
\end{aligned}
$$

where

$$
\tilde{T}_{\mu \nu}=\frac{2}{\sqrt{-\tilde{g}}} \frac{\delta S_{m}}{\delta \tilde{g}^{\mu \nu}},
$$

is the energy-momentum tensor of the matter content and $\tilde{T} \equiv \tilde{T}_{\mu}^{\mu}$ is its trace. Clearly, there is an equivalence between the scalar-tensor action (2.1) and the general relativity case, though this feature is not generally true. If $\tilde{T}$ vanishes and $\tilde{\Phi}$ is a constant, equations (2.2) reduce to the usual Einstein's equations if we identify a gravitational constant $G^{*}$ with the inverse of the scalar field, e.g., $G^{*}=1 / \tilde{\Phi}$. Hence, any exact solution of Einstein's equations with a trace-free matter source will also be a particular exact solution of the scalar-field with $\tilde{\Phi}$ constant. Of course, this particular solution will not be the general solution for the matter content. En passant, we mention that, when the scalar field kinetic term is absent in (2.1), these theories are equivalent to the $1 / R$ gravity theories. In this case, the scalar field is not an independent dynamical degree of freedom and, among many consequences, these theories are in conflict with solar system observations [15] and are not suitable to describe the cosmic acceleration [16].

If we make a conformal deformation

$$
\tilde{g}_{\mu v}=A^{2}(\phi) g_{\mu v}
$$

and a redefinition of the quantities

$$
G^{*} A^{2}(\phi)=\frac{1}{\tilde{\Phi}}
$$

$G^{*}$ is a "bare" gravitational constant, and

$$
\alpha(\phi)=\frac{\partial \ln A(\phi)}{\partial \phi}=\frac{1}{(2 \omega(\tilde{\Phi})+3)^{1 / 2}},
$$


which can be interpreted as the (field-dependent) coupling strength between matter and scalar field, then (2.1) can be re-written in the Einstein (also called conformal) frame in which the kinematic terms of tensor and scalar do not mix:

$$
\mathcal{S}=\frac{1}{16 \pi G^{*}} \int \sqrt{g}\left[R-2 g^{\mu v} \partial_{\mu} \phi \partial_{v} \phi\right]+\mathcal{S}_{m}\left[\Psi_{m}, A^{2}(\phi) g_{\mu v}\right],
$$

where $g_{\mu v}$ is a pure rank-2 metric tensor and $R$ is the curvature scalar associated to it.

In the conformal frame, eqs. (2.2) are written in a more convenient form:

$$
\begin{aligned}
R_{\mu \nu}-\frac{1}{2} g_{\mu \nu} R & =8 \pi G^{*} T_{\mu \nu}+2 \partial_{\mu} \phi \partial_{\nu} \phi-g_{\mu \nu} g^{\alpha \beta} \partial_{\alpha} \phi \partial_{\beta} \phi \\
\square_{g} \phi & =-4 \pi G^{*} \alpha(\phi) T .
\end{aligned}
$$

It has been shown in [11] that we can relate the metric in the scalar-tensor gravities with the metric in Einstein's gravity for the same matter distribution in the weak-field approximation regime. For a matter of consistency, we will summarize this method below.

Let us expand the fields to first order in the parameter $G_{0}=G^{*} A^{2}\left(\phi_{0}\right)$, we then obtain

$$
\begin{aligned}
g_{\mu v} & =\eta_{\mu v}+h_{\mu v}, \\
\phi & =\phi_{0}+\phi_{(1)}, \\
A(\phi) & =A\left(\phi_{0}\right)\left[1+\alpha\left(\phi_{0}\right) \phi_{(1)}\right], \\
T_{v}^{\mu} & =T_{(0) v}^{\mu}+T_{(1) v}^{\mu} .
\end{aligned}
$$

Therefore, eqs. (2.8) reduce to:

$$
\begin{aligned}
\nabla^{2} h_{\mu v} & =16 \pi G^{*}\left(T_{\mu v}^{(0)}-\frac{1}{2} \eta_{\mu v} T_{(0)}\right), \\
\nabla^{2} \phi_{(1)} & =4 \pi G^{*} \alpha\left(\phi_{0}\right) T_{(0)} .
\end{aligned}
$$

In this approximation, $T_{\mu \nu}^{(0)}$ is the energy-momentum tensor at zeroth-order in the conformal frame. Its relation to the (physical) energy-momentum tensor at zeroth-order in the Jordan-Fierz frame is given by $T_{\mu \nu}^{(0)}=A^{2}\left(\phi_{0}\right) \tilde{T}_{\mu \nu}^{(0)}$. In this way, the first equation in the system (2.10) is the Einstein's equation in the weak-field approximation regime.

Once we have $\tilde{g}_{\mu v}=A^{2}(\phi)\left[\eta_{\mu v}+h_{\mu v}\right]$, we can then use the approximation (2.9). We obtain:

$$
\tilde{g}_{\mu v}=A^{2}\left(\phi_{0}\right)\left[1+2 \alpha\left(\phi_{0}\right) \phi_{(1)}\right]\left[\eta_{\mu v}+h_{\mu v}\right] .
$$

We can apply now this algorithm to the case of the chiral string. In the weak field approximation, the method described above is, schematically

$$
d s_{S T}^{2}=\left[1+2 \alpha\left(\phi_{0}\right) \phi_{(1)}\right] d s_{G R}^{2},
$$

where $d s_{S T}^{2}$ is the line element in the scalar-tensor theory, and $d s_{G R}^{2}$ is the line element in general relativity and $\phi_{(1)}$ is the solution of the equation

$$
\nabla^{2} \phi_{(1)}=4 \pi G^{*} \alpha\left(\phi_{0}\right) T_{(0)}
$$


In the ref. [17], the metric and the energy-momentum tensor for the chiral cosmic string in general relativity for the weak field regime were obtained:

$$
\begin{aligned}
d s^{2} & =d t^{2}[1+X(r, k)]-d z^{2}[1-X(r, k)]-d r^{2} \\
& +\left[1-4 G^{*} m^{2}\right]^{2} r^{2} d \theta^{2}-2 X(r, k) d t d z
\end{aligned}
$$

where $0 \leq k \leq 1$ is the chiral factor which characterizes the state of the string: $k=0$ corresponds to the maximal charged strings and $k=1$ corresponds to the ordinary, neutral string. It can happen that $k$ is not constant, but we will not deal with this case here,

$$
T_{(0)}^{\mu \nu}=\frac{2 m^{2}}{1+k}\left(\begin{array}{cccc}
1 & 0 & 0 & \frac{1-k}{2} \\
0 & 0 & 0 & 0 \\
0 & 0 & 0 & 0 \\
\frac{1-k}{2} & 0 & 0 & -k
\end{array}\right) \delta(x) \delta(y)
$$

where $X(r, k)=8 G^{*} m^{2} \frac{1-k}{1+k} \ln \left(\frac{r}{r_{0}}\right)$. With this, we find the trace of the energy-momentum tensor $\left(T_{(0)}=2 A^{2}\left(\phi_{0}\right) m^{2} \delta(x) \delta(y)\right)$. Using the result $\nabla^{2} \ln \left(\frac{r}{r_{0}}\right)=2 \pi \delta(x) \delta(y)$ we have

$$
\nabla^{2} \phi_{(1)}=8 \pi m^{2} G_{0} \alpha\left(\phi_{0}\right) \delta(x) \delta(y) \rightarrow \phi_{(1)}=4 m^{2} G_{0} \alpha\left(\phi_{0}\right) \ln \left(\frac{r}{r_{0}}\right) .
$$

Therefore, the metric for the scalar-tensor chiral string is, to first order in $G_{0}$

$$
\begin{aligned}
d s_{S T}^{2} & =\left(1+8 \alpha\left(\phi_{0}\right)^{2} m^{2} G_{0} \ln \left(\frac{r}{r_{0}}\right)\right)\left[\left(1+8 G_{0} m^{2} \frac{1-k}{1+k} \ln \left(\frac{r}{r_{0}}\right)\right) d t^{2}\right. \\
& -d r^{2}-\left(1-4 G_{0} m^{2}\right) r^{2} d \theta^{2}-\left(1-8 G_{0} m^{2} \frac{1-k}{1+k} \ln \left(\frac{r}{r_{0}}\right)\right) d z^{2} \\
& \left.-2\left(8 G_{0} m^{2} \frac{1-k}{1+k} \ln \left(\frac{r}{r_{0}}\right)\right) d z d t\right] .
\end{aligned}
$$

Consequently, associated with the metric (2.15), we have a gravitational force acting on a test particle of mass $M$ :

$$
F=-\frac{4 M}{r}\left(\alpha\left(\phi_{0}\right)^{2} m^{2}+\left(\frac{1-k}{1+k}\right) m^{2}\right) G_{0}
$$

\subsection{The Zel'dovich approximation and the accretion problem}

To determine the displacement of a particle flowing past a cosmic string to study in the next section the formation and evolution of wakes, we will use the Zel'dovich approximation [18]. To start with, we first compute the velocity perturbation of massive particles moving past the string. If we consider that the string is moving with normal velocity $v_{s}$ through matter, the velocity perturbation can be calculated ${ }^{3}$ here with the help of the gravitational force due to metric (2.15):

$$
u_{i}(r)=8 \pi G_{0} m^{2} v_{s} \gamma+\frac{4 \pi G_{0} \alpha\left(\phi_{0}\right)^{2} m^{2}}{v_{s} \gamma}+\left(\frac{1-k}{1+k}\right) \frac{4 \pi G_{0} m^{2}}{v_{s} \gamma}
$$

\footnotetext{
${ }^{3}$ All expansions in the parameter $G_{0}$ were performed using the algebraic computational programme MAPLE.
} 
with $\gamma=\left(1-v_{s}^{2}\right)^{-1 / 2}$. The first term in (2.16) is equivalent to the relative velocity of particles flowing past a string in general relativity. The other terms come as a consequence of the scalartensor coupling of the gravitational interaction and the chirality properties of the string.

Let us suppose now that the wake was formed at $t_{i}>t_{e q}$. The physical trajectory of a dark particle can be written as

$$
h(\vec{x}, t)=a(t)[\vec{x}+\psi(\vec{x}, t)],
$$

where $\vec{x}$ is the unperturbed comoving position of the particle and $\psi(\vec{x}, t)$ is the comoving displacement developed as a consequence of the gravitational attraction induced by the wake on the particle. Suppose, for simplification, that the wake is perpendicular to the $x$-axis (assuming that $d z=0$ in the metric (2.15) and $r=\sqrt{x^{2}+y^{2}}$ ) in such a way that the only non-vanishing component of $\psi$ is $\psi_{x}$. Therefore, the equation of motion for a dark particle in the Newtonian limit is

$$
\ddot{h}=-\nabla_{h} \Phi,
$$

where the Newtonian potential $\Phi$ satisfies the Poisson equation

$$
\nabla_{h}^{2} \Phi=4 \pi G_{0} \rho
$$

where $\rho(t)$ is the dark matter density in a cold dark matter universe. For a flat universe in the matter-dominated era, $a(t) \sim t^{2 / 3}$. Therefore, the linearised equation for $\psi_{x}$ is

$$
\ddot{\psi}+\frac{4}{3 t} \dot{\psi}-\frac{2}{3 t^{2}} \psi=0,
$$

with appropriated initial conditions: $\psi\left(t_{i}\right)=0$ and $\psi\left(t_{i}\right)=-u_{i}$. Eq. (2.20) is the Euler equation whose solution is easily found

$$
\psi(x, t)=\frac{3}{5}\left[\frac{u_{i} t_{i}^{2}}{t}-u_{i} t_{i}\left(\frac{t}{t_{i}}\right)^{2 / 3}\right] .
$$

Calculating the comoving coordinate $x(t)$ using the fact that $\dot{h}=0$ in the "turn around" 4 , we get

$$
x(t)=-\frac{6}{5}\left[\frac{u_{i} t_{i}^{2}}{t}-u_{i} t_{i}\left(\frac{t}{t_{i}}\right)^{2 / 3}\right] .
$$

The thickness and the wake's superficial density are, respectively:

$$
\begin{aligned}
d(t) & \approx 2 x(t)\left(\frac{t}{t_{i}}\right)^{\frac{2}{3}}=\frac{12}{5} u_{i} t_{i}\left(\frac{t^{\frac{4}{3}}}{t_{i}^{\frac{4}{3}}}\right)=\frac{12}{5}\left(8 \pi G_{0} m^{2} v_{s} \gamma\right. \\
& \left.+\frac{4 \pi G_{0} \alpha\left(\phi_{0}\right)^{2} m^{2}}{v_{s} \gamma}+\left(\frac{1-k}{1+k}\right) \frac{4 \pi G_{0} m^{2}}{v_{s} \gamma}\right),
\end{aligned}
$$

and

$$
\begin{aligned}
\sigma(t) & \approx \rho(t) d(t)=\frac{2 u_{i}}{5 \pi G_{0} t}\left(\frac{t}{t_{i}}\right)^{\frac{1}{3}} \\
& =\left(\frac{16}{5} \frac{m^{2} v_{s} \gamma}{t}+\frac{8}{5} \frac{\alpha\left(\phi_{0}\right)^{2} m^{2}}{v_{s} \gamma t}+\frac{8}{5}\left(\frac{1-k}{1+k}\right) \frac{m^{2}}{v_{s} \gamma t}\right)\left(\frac{t}{t_{i}}\right)^{\frac{1}{3}} .
\end{aligned}
$$

We summarize our results in the next section.

\footnotetext{
${ }^{4}$ The moment when the dark particle stops expanding with the Hubble flow and starts to collapse onto the wake.
} 


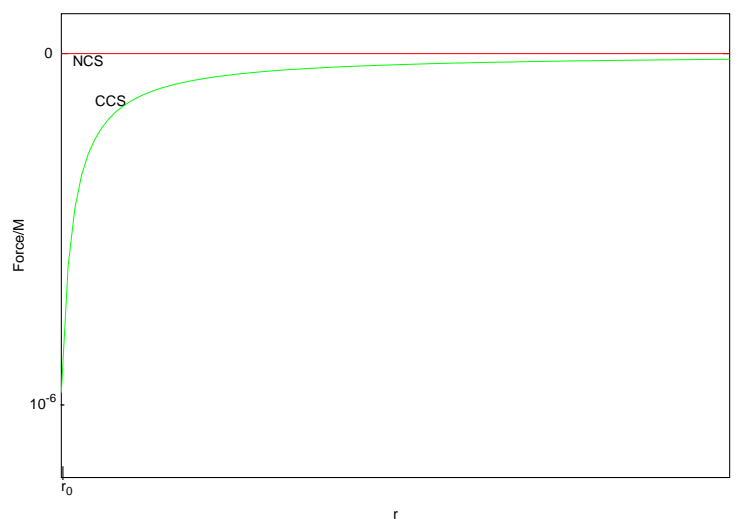

Figure 1: Gravitational force for a neutral (NCS) and chiral strings (CCS) in general relativity

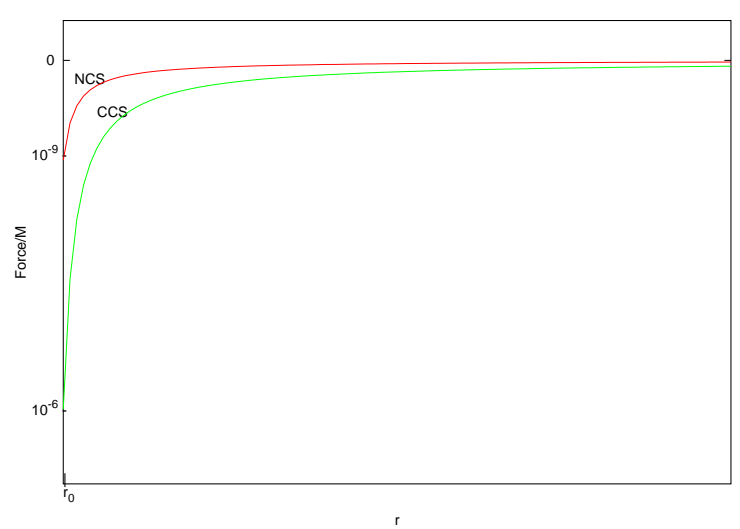

Figure 2: Gravitational force for a neutral (NCS) and chiral (CCS) strings in scalar-tensor theories

\section{Main results}

Inclusion of a current in the internal structure of a cosmic vortice can drastically change the predictions of such models in a number of small and large-scale effects. In particular, models with a timelike-type or a spacelike-type currents can present divergences leading to unbounded gravitational effects [8, 9].

In this work, we have concentrated on the chiral string model for which the world-sheet current is null in the framework of a scalar-tensor gravity. Our main goal was to analyse the impact of such a current on the gravitational macroscopic effects. For the purpose of this analysis, we first studied the gravitational properties of the spacetime generated by this string in the presence of a dilaton field. Then, we carried out an investigation of the mechanism of formation and evolution of wakes in this framework, showing the explicit contribution of the chirality to this effect.

Surprisingly enough, the mechanism of accretion is not sensitive to the "conducting properties" of this model, the results being qualitatively very similar to the mechanism by a neutral string, up to first order in the parameter $G_{0}$. The divergences appear at higher orders in this parameter.

\section{Comparison with other cosmic string models}

- Chiral strings in general relativity and scalar-tensor theories 
It is interesting to observe that chiral string models behave qualitatively in the same way for both theories of gravity. In the weak-field approximation, which is indeed the regime under consideration, the scalar-tensor model has an additional factor of order $10^{3}$ smaller than his general relativity partner. This factor is introduced by the post-Newtonian parameter $\alpha_{0}$ which expresses the strength of the interaction between matter and the dilaton field.

\section{- Chiral and neutral strings in general relativity and scalar-tensor theories}

To introduce chirality and dilatonic properties in a cosmic string model definitely changes the behavior of the gravitational force on test particles, as can be seen from the Figs. 1 and 2. Nevertheless, the impact on the formation of wakes is neglegible to first order in the parameter $G_{0}$.

\section{Acknowledgements}

M. E. X. Guimarães would like to thank the Conselho Nacional de Desenvolvimento Científico e Tecnológico (CNPq) for a support and A. L. Naves de Oliveira would like to thank the Coordenação de Aperfeiçoamento de Pessoal de Nível Superior (CAPES/MEC) for a $\mathrm{PhD}$ grant. The authors would like to thank FINATEC (UnB) for partial support.

\section{References}

[1] E. Witten, Superconducting Strings, Nucl. Phys. B 249 (1985) 557.

[2] N. Arkani-Hamed, S. Dimopoulos and G. R. Dvali, The Hierarchy Problem and New Dimensions at a Millimiter, Phys. Lett. B429 (1998) 263 [hep-ph/9803315].

N. Arkani-Hamed, S. Dimopoulos and G. R. Dvali, Phenomenology, Astrophysics and Cosmology of Theories with Sub-millimeter Dimensions and TeV Scale Quantum Gravity, Phys. Rev. D 59 (1999) 086004 [hep-ph/9807344].

[3] L. Randall and R. Sundrum, A Large Mass Hierarchy from a Small Extra Dimension, Phys. Rev. Lett. 83 (1999) 3370 [hep-ph/9905221].

[4] Y. Fujii and K. Maeda, The Scalar-Tensor Theory of Gravitation, Cambridge Univ. Press, Cambridge 2003.

[5] S. Nussinov and R. Shrock, Some Remarks on Theories with Large Compact Dimensions and TeV-scale Quantum Gravity, Phys. Rev. D 59 (1999) 105002 [hep-ph/9811323].

[6] M. Milgrom, A Modification of the Newtonian Dynamics as a Possible Alternative to the Hidden Mass Hypothesis, Ap. J. 270 (1983) 365.

[7] C. N. Ferreira, M. E. X. Guimarães and J. A. Helayël-Neto, Current Carrying Cosmic Strings in Scalar Tensor Gravities, Nucl. Phys. B 581 (2002) 165 [gr-qc/ 0002054 ];

V. B. Bezerra and C. N. Ferreira, Gravitational Field Around a Screwed Superconducting Cosmic String in Scalar-Tensor Theories, Phys. Rev. D 65 (2002) 084030 [hep-th/ 0111167].

[8] A. L. N. Oliveira and M. E. X. Guimarães, Wakes in Dilatonic Current-Carrying Cosmic Strings, Phys. Rev. D 67, 123514 (2003) [hep-th/0303112].

[9] A. L. N. Oliveira and M. E. X. Guimarães, Breakdown of the Mechanism of Forming Wakes by a Current-Carrying String, Phys. Lett. A 311 (2003) 474 [hep-th/ 0304242$].$ 
[10] P. Peter, M. E. X. Guimarães and V. C. Andrade, Dilatonic Current-Carrying Cosmic Strings, Phys. Rev. D 67 (2003) 123509 [gr-qc/ 0101039 ].

[11] M. E. X. Guimarães, L. P. Colatto and F. B. Tourinho, On the Weak Field Approximation in Generalized Scalar Tensor Gravities, Spacetime and Substance 2 (2001) 71 [gr-qc/0201101].

[12] A. Stebbins, A. Veeraraghavan, R.H. Brandenberger, J. Silk, N. Turok Cosmic Strings Wakes, Ap. J. 322 (1987) 1;

T. Vachaspati, Structure of Wiggly-Cosmic-String Wakes, Phys. Rev. D 45 (1992) 3487.

[13] S. R. M. Masalskiene and M. E. X. Guimarães, Cosmic String Wakes in Scalar-Tensor Gravities, Class. Quant. Grav. 17 (2000) 3055 [gr-qc/ 0003041$].$

[14] M. C. B. Abdalla, A. A. Bytsenko and M. E. X. Guimarães, Formation of Wakes by Chiral, Conducting Cosmic Strings, Modern Phys. Lett. A 19 (2004) 2445.

[15] T. Chiba, 1/R Gravity and Scalar-Tensor Gravity, Phys. Lett. B 575 (2003) 1 [astro-ph/0307338].

[16] E. A. Flanagan, Higher Order Gravity Theories and Scalar Tensor Theories, Class. Quantum Grav. 21 (2003) 417 [gr-qc/ 0309015$]$;

E. A. Flanagan, Palatini Form of 1/R Gravity, Phys. Rev. Lett. 92 (2004) 071101 [astro-ph/0308111].

[17] D. A. Steer, Self-Interactions and Gravitational Properties of Chiral Cosmic Strings in Minkowski Space, Phys. Rev. D 63 (2001) 083517 [astro-ph/0 011233 ].

[18] Ya. B. Zel'dovich, Gravitational Instability: An Approximate Theory for Large Density Perturbations, Astron. Ap. 5 (1970) 84. 